\title{
Organismos internacionales y gobernanza regional: una policy transfer para la educación de la infancia en países de América Latina y el Caribe
}

\author{
Camila Maria Bortot (D) @ \\ Elisângela Alves da Silva Scaff (D) @ \\ Universidade Federal do Paraná (UFPR), Brasil
}

Resumen: El artículo analiza el papel de los organismos internacionales (OI) como órganos de gobernanza regional para la creación e institucionalización de una agenda intersectorial de la infancia en América Latina y el Caribe. Se realizó por medio de una investigación bibliográfica y documental, apropiándose en el abordaje teórico de un concepto del campo de la Ciencia Política denominado policy transfer. Se mapearon experiencias latinoamericanas y caribeñas que involucrasen acciones intersectoriales; se identificó como modelo de éxito para la educación de la infancia, el programa cubano Educa a tu Hijo, transferido a Ecuador, Brasil, México, Colombia y Guatemala a través de los OI. Se observó que la institucionalización de ese modelo, mediado por las agencias internacionales por la vía de la gobernanza negociada y facilitado con los Estados nacionales, ocurrió de formas diferentes en los países involucrados. Entre los principales cambios verificados en la transferencia de esa política, se registraron: descentralización; programas de bajo costo; atención no formal como alternativa a la escolar; agenda internacional, flexibilización; carácter compensatorio; alianzas público-privadas para la atención; y, discontinuidades en el desarrollo de la política en municipios de algunos países. Palabras clave: gobernanza regional; organismos internacionales; políticas Intersectoriales para la Infancia; policy transfer.

\begin{abstract}
Organismos internacionais e governança regional: uma policy transfer para a Educação Infantil em países da América Latina e do Caribe

Resumo: Neste artigo pretende-se analisar o papel dos organismos internacionais (OI) como órgãos de governança regional para a criação e institucionalização de uma agenda intersetorial para a infância na América Latina e no Caribe. O estudo foi realizado mediante uma pesquisa bibliográfica e documental que privilegiou a abordagem teórica do campo da Ciência Política denominado policy transfer. No princípio, foi feito um mapeamento das experiências latino-americanas e caribenhas que envolvessem ações intersetoriais, determinando como modelo de sucesso para a educação infantil o programa cubano Eduque seu filho, transferido ao Equador, Brasil, México, Colômbia e à Guatemala com a mediação dos Ol. Pôde-se observar que a institucionalização desse modelo, mesmo sendo mediada pelas agências internacionais pela via da governança negociada e facilitada com os Estados nacionais, ocorreu de formas diferentes nos diversos países envolvidos. Entre as principais mudanças verificadas na transferência dessa política estão: descentralização; programas de baixo custo; atendimento não formal como alternativa ao escolar; agenda internacional, flexibilização; caráter compensatório; parcerias público-privadas para o atendimento, descontinuidades no desenvolvimento da política em municípios.
\end{abstract}

Palavras-chave: governança regional; organismos internacionais; políticas intersetoriais para a infância; policy transfer.

\section{International organizations and regional governance: a policy trasnfer for early childhood edu- cation in Latin American and Caribbean countries.}

Abstract. This article seeks to analyze the role of International Organizations as regional governance agency for the creation and institutionalization of an intersectorial schedule for children in Latin America and the Caribbean. The study was carried out through bibliographic and documentary research, privileging the theoretical approach of the field of Political Science, called policy transfer. Initially, a mapping of Latin American and Caribbean experiences involving intersectoral actions was carried out, identifying the Cuban Educa a tu hijo program as a model of success for childhood education, transferred to Ecuador, Brazil, Mexico, Colombia and Guatemala, under the mediation of IOs. It was observed that the institutionalization of this model, although mediated by international agencies, through governance negotiated and facilitated with national states, occurred in different ways in the different countries involved. Among the main changes in the transfer of this policy are: decentralization; low-cost programs; non-formal care as an alternative to school; international schedule; flexibility; compensatory character; public-private partnerships for service; discontinuities in policy development in municipalities in some countries.

Keywords: regionalgovernance; internationalorganizations; intersectoral policies forchildren; policy transfer. 


\section{Introducción}

En este artículo se busca analizar el papel de los organismos internacionales (OI) como órganos de gobernanza regional en la creación e institucionalización de una agenda intersectorial para la infancia en América Latina y el Caribe (ALC).

La agudización de las contradicciones sociales, con el correlativo crecimiento de las desigualdades, que se expresan en los elevados índices de aumento de la pobreza y del desempleo en la región latinoamericana, hizo que, en la década de los 90, gobiernos y Ol como el Banco Mundial, la Comisión Económica para América Latina y el Caribe (Cepal), la Organización de las Naciones Unidas para la Educación, la Ciencia y la Cultura (Unesco) y el Fondo de las Naciones Unidas para la Infancia (Unicef) empezaran a presentar propuestas a favor de un amplio ajuste económico y social. El desencadenamiento de reuniones y la puesta en marcha de diversas acciones y programas, a veces financiados y diseñados por los OI, se concentraron para el reposicionamiento de la educación en el marco de las estrategias de desarrollo como una política prioritaria (Casassus, 2001), estimulando programas de educación, integrados a programas intersectoriales de atención familiar.

En general, mecanismos de coordinación intersectorial para la atención a la educación para la infancia, impulsados por iniciativas internacionales como la Convención sobre los Derechos del Niño (1989) y la Cumbre Mundial en favor de la Infancia (1990), en las que los Estados asumieron en parte ${ }^{1}$ la obligación de desarrollar políticas comprometidas en la protección de los derechos de los niños (López y D’Alessandre, 2015; Bortot y Lara, 2019), este período generó un terreno fértil de reuniones y acuerdos para la educación de la infancia entre los Estados nacionales y los OI, sobre todo por la Unesco y la Unicef, en el planeamiento de las acciones instituidas, así como en la evaluación y divulgación de prácticas exitosas que lograsen los objetivos de estos compromisos, para implementar algunas de estas estrategias en la región.

Se publicaron estudios de la Unesco que contextualizaron el panorama latinoamericano y caribeño sobre las acciones instituidas en Jontiem, Dakar y Cochabamba, resultando documentos como: Participación de Las Familias en La Educación Infantil Latinoamericana (Unesco, 2004) y Síntesis Regional de Indicadores de La Primera Infancia (Blanco y Umayahara, 2004). Un análisis, llevado a cabo por Bortot (2018), pone de manifiesto que las orientaciones contenidas en dichos documentos, señalan una dicotomía entre la franja etaria de 0-3 años y la de 4-6 años. En función de cada una,

${ }^{1}$ Sobre la ratificación de la Convención por parte de los países en cuanto a las acciones instituidas en 1989, ver Bortot y Lara (2019). 
la base intersectorial se debe realizar por medio de una división de tareas en la gestión de los sistemas educativos, ya que la provisión de la oferta en los niveles considerados "formales" queda bajo la responsabilidad públicoestatal, y la modalidad "no formal", por ser considerada "no escolar", tiene una formación compensatoria, que depende principalmente de programas, acuerdos 0 asociaciones público-privadas, con el fin de atender a otros niños no asistidos (Bortot, 2018).

Así, la educación intersectorial de la infancia debe realizarse como una estrategia de gobernanza, a través de la comunidad educativa y de los más variados acuerdos institucionales, denominados como "[...] nuevos tipos de actores sociales, sujetos sociales híbridos que son espacialmente móviles, éticamente maleables y capaces de hablar los lenguajes de lo público, del valor privado y filantrópico" (Ball, 2014, p. 230). En dicho contexto, las políticas educativas están mayormente orientadas a partir de prácticas de gobernanza (Unesco, 2002b; 2007), tomando como referencia programas intersectoriales considerados exitosos (Unesco, 2004; Blanco y Umayahara, 2004), en especial, para el desarrollo de la educación y cuidado de la primera infancia, habida cuenta de que, al mismo tiempo, se actúa en la gestión de la pobreza. La gobernanza regional se realiza a partir de acciones de cooperación internacional, con la suma de esfuerzos de asociaciones vinculadas al Tercer Sector, por medio de programas que aporten a la comunidad educativa.

El programa intersectorial, entendido como estrategia de gobernanza regional, también se desarrolló por medio de técnicas de producción de la política educativa, cuya agenda se materializa "[...] como préstamo, convergencia y transferencia de políticas y se acomoda a las especificidades históricas y culturales de cada país" (Costa, 2019, p. 8), mediadas por agentes de transferencia como los OI (Stone, 2004; Gorostiaga, 2020; Shiroma, 2020).

Los documentos producidos para la infancia en el umbral del siglo XXI, facilitaron la proyección de una agenda de la política educativa intersectorial, dando inicio a la materialización de la agenda para Latinoamérica y el Caribe. Considerando ese escenario, en el presente texto se busca responder a estas cuestiones: ¿de qué manera los OI realizan la gobernanza de las acciones intersectoriales para la educación de la infancia en países de ALC?, ¿qué acciones se han priorizado en ese contexto?, ¿cómo los países se apropian de las acciones intersectoriales en sus políticas? Abstrayéndose de la dimensión del contexto regional a partir del problema aquí planteado, se busca develar las confluencias y divergencias en sus múltiples determinaciones.

La investigación está fundamentada en un abordaje cualitativo y se llevó a cabo a partir de un relevamiento bibliográfico y documental. La selección de las fuentes se basó en los siguientes criterios: que la temáti- 
ca tratase de la intersectorialidad para la infancia; $y$, haber sido publicado entre la última década del siglo XX y la primera década del siglo XXI, lo que favorece la comparación con las recomendaciones internacionales y políticas incorporadas por los países.

La recogida de documentos se realizó en los sitios oficiales de los Estados nacionales y de los Ol. También se basó en la policy transfer, abordaje teórico del campo de la Ciencia Política, definido por Cowen (2006) como un movimiento de ideas, políticas y prácticas que se desplazan de un lugar a otro, generalmente, entre fronteras nacionales. A veces, este fenómeno es el resultado de programas de cooperación multilateral, establecidos con los gobiernos. Estos, denominados receptores, pueden no desear el contenido de la transferencia, pero se sienten obligados a aceptarlo para poder recibir otros beneficios (Shiroma, 2020), pues son signatarios de la agenda de decisiones político-educativas transnacionales (Krawczyk, 2019).

En tal sentido, los análisis se concentraron en las instituciones más participativas -como la Unesco y la Unicef- para la creación de una agenda político-educativa y en la transferencia de políticas para la infancia. La elección de estas agencias internacionales fue el resultado de su tenaz movilización de esfuerzos y de su poder de convocatoria para la efectividad del/en el programa intersectorial (Umayahara, 2004):

[...] a distintos sectores y actores, [por] tener acceso a las altas autoridades gubernamentales y ejercer presión sobre los gobiernos en función de acuerdos y compromisos internacionales (p. 93).

El texto se desarrolla en dos partes, en la primera se aborda cómo la intersectorialidad es admitida como una estrategia de gobernanza regional a través de los OI en tiempos neoliberales; en la segunda, se analiza cómo se incorpora un programa exitoso a partir de la policy transfer, en virtud de los cuales los OI se convierten en agentes en la práctica de gobernanza regional. Por último, hilvanamos las consideraciones y reflexiones finales.

\section{La educación intersectorial de la infancia como estrategia de gobernanza}

Las tres últimas décadas del siglo XX fueron prolíficas en reformas estructurales en diversos países del mundo, teniendo como precursores a Inglaterra y a los Estados Unidos de América. La alternativa que esos países encontraron para la superación de la crisis económica resultó, directamente, en un nuevo ordenamiento de la forma de gestión del Estado, posicionando al 
mercado como modelo de eficiencia en la oferta de servicios públicos (Scaff, 2017). Este modelo, denominado Estado gerencial, encontró su fundamento en las teorías posburocráticas, que lo proponían como alternativa a la considerada vieja, cara e ineficiente burocracia. Esta perspectiva de la gestión gerencial, como referencia de gobernabilidad del Estado, pasó a incorporar un conjunto de principios de la gestión privada con vistas a la optimización de los servicios en el ámbito del sector público (Verger y Normand, 2015).

La teoría de la gobernanza surge, así, como alternativa al modelo de gerencialismo angloamericano (Casula, 2017). Aunque aún requiera mayor precisión conceptual, se puede considerar que la gobernanza implica la redefinición de un Estado activo, productor del bien público, como un Estado activador "que acciona y coordina otros actores para que produzcan con él" (Kissler y Heidemann, 2006, p. 483). En esa configuración, "Estado, mercado, redes sociales y comunidades constituyen mecanismos institucionales de regulación que se articulan en diferentes composiciones o acuerdos" ( $p$. 486). En la esfera internacional, el concepto de gobernanza global incorpora nuevos actores no estatales, como corporaciones internacionales, ONG, representantes del sector privado y de la sociedad civil, que intervienen en la toma de decisiones (Casula, 2017; Pereira, 2019).

La gobernanza global puede definirse como un sistema de valores, políticas e instituciones que posibilitan a las sociedades organizarse para tomar decisiones colectivas y ejercer acciones de orden político, económico, sociocultural y ambiental por medio de la interacción entre Estado, sociedad civil y sector privado. Este proceso se puede realizar de diferentes formas: acciones estatales unilaterales en el propio territorio; acciones unilaterales fuera de los Estados; cooperación regional y multilateral por medio de la creación de regímenes internacionales; y, cooperación transnacional y transgubernamental, reconociendo que la conexión entre el Estado y la sociedad importa (Rhodes, 2012).

En este artículo, vemos a los OI como actores fundamentales en las relaciones de gobernanza establecidas en $\mathrm{ALC}$, considerando el avance del proyecto político transnacional en los últimos años, que ha provocado el fortalecimiento de la actividad de elaboración de políticas globales. En ese proyecto regional/ transnacional, los $\mathrm{OI}$ se han vuelto protagonistas en la elaboración de acuerdos y programas, actuando como agentes de mediación y cooperación internacional, especialmente tras el Consenso de Washington ${ }^{2}$ a finales de los años 80 , cuyas

${ }^{2}$ Se conoce como Consenso de Washington a un conjunto de diez recomendaciones de política económica formuladas en 1989 por el economista inglés John Williamson, que tenían como objetivo orientar a los países en desarrollo inmersos en la crisis económica para que lograsen salir de la misma. 
orientaciones desencadenaron una generación de préstamos condicionados a la implantación de reformas de ajuste estructural, como la del Banco Mundial en la educación brasileña (Ferreira, Fonseca y Scaff, 2019).

\section{En el Cuadro 1, presentamos las etapas que configuran la agenda social en Latinoamérica en el período contemplado por la investigación.}

\section{Cuadro 1. Etapas de la agenda social en América Latina (1970-1990)}

\begin{tabular}{lll}
\hline Período & $\begin{array}{l}\text { Principales } \\
\text { elementos }\end{array}$ & Características \\
\end{tabular}

1970 Cambio de los regíme- Diseminación y aceptación de los valores republicanos

1980 nes autoritarios (de igualdad, individualismo, derechos sociales, individuales y colectivos); fuerte competitividad electoral, alta participación política por medio del restablecimiento de las libertades democráticas y del Estado de Derecho.

1980 Globalización: ajustes A pesar de la nueva institucionalidad en esos países, el 1990 fiscales; estabiliza- impacto de las tendencias internacionalizadas de políticas ción monetaria neoliberales, internamente, de forma no homogénea, implicó la reconversión de los principios democráticos en la defensa del "libre" mercado, traducida por los ajustes fiscales y estabilización monetaria, reestructuración productiva, privatizaciones de servicios y empresas públicas, descentralización de políticas y desconcentración por medio de procesos de Reforma del Estado.

1990 Actores internacionales; reestructuración productiva; aumento de la pobreza
Acuerdos en el ámbito de la determinación de actores internacionales (Comisiones multilaterales) bajo la hegemonía del mercado y legitimidad otorgada por la estabilidad monetaria, que restringe la agenda del Ejecutivo en un contexto de vigencia democrática a la puesta en marcha de ajustes y gestión institucional organizados en torno a principios normativos y morales del "buen gobierno" para la eficiencia técnica y ajuste monetario de las cuentas públicas. Sin embargo, dicha hegemonía se expresa en el contrapunto de los efectos perversos de la reestructuración productiva de los ajustes del Estado sobre la sociedad, definiéndose ahora a partir de una responsabilidad social frente "a sus efectos", a saber, el enfrentamiento y superación de la pobreza como condición política compensatoria de integración social, sustitutiva de los dispositivos del Estado social y responsabilidad del conjunto de la sociedad. Por medio de una "retórica" y ética moral de compromiso en el enfrentamiento de las desigualdades sociales, se desmonta la estructura de los derechos y la responsabilidad pública del Estado frente a la cuestión social, que queda subordinada a la filantropía y a la gestión de metodologías y políticas de focalización.

Fuente: adaptado por las autoras a partir de Ivo (2002). 
Habida cuenta del endeudamiento de los países de Latinoamérica, las agencias internacionales efectuaron un proceso de nuevas orientaciones políticas, basadas en un conjunto de reformas fundadas en el Consenso de Washington. Los mercados, la competencia y el papel del sector privado en nuevas y viejas áreas de servicios, incluyendo la educación, se presentaron como "de interés nacional" (Ball, 2007; 2014), centrales para la competitividad en la economía global, como medio de disminuir la pobreza y estancar la desaceleración del crecimiento económico y como fundamentos para la construcción de economías basadas en el conocimiento (Harvey, 2008; Robertson y Verger, 2012; Robertson y Dale, 2017).

De forma regional, la gobernanza fue construida en su núcleo estructural por esferas de acción política en distintos niveles, especialmente subnacional, nacional y supranacional, con la emergencia de actores de la sociedad civil y no gubernamentales. Ese modelo exigió una reconfiguración conceptual multidimensional, cimentada por dinámicas más globales que privilegiaron la inclusión y la progresiva relación entre los actores del sistema internacional.

Las reformas estructurales en la gobernanza, fomentadas por los Ol, pusieron en jaque el modelo de gobernación estatal, buscando, a partir del discurso de la modernización, favorecer pactos más globales y políticas públicas más transversales, específicamente en la educación, la salud, los derechos humanos, el medio ambiente y la economía.

La focalización de estas reformas tuvo a la pobreza como objeto, constituyéndose en uno de los pilares, especialmente en los países de Latinoamérica. Aunque no sea un fenómeno único de ese período en la región, la pobreza adquirió mayor consistencia a partir de las crisis económicas que afectaron a la mayoría de los países en las décadas de 1970 y 1980 . En ese escenario, la educación de la infancia se concibe a partir del entendimiento de combatir a la pobreza y pasa a integrar la agenda política de desarrollo económico y social, elaborada por las agencias vinculadas a las organizaciones de las Naciones Unidas.

En los años 1990 y comienzos de los años 2000, se produjeron muchos documentos destinados a orientar las políticas de intersectorialidad para la infancia. Entre ellos, cabe resaltar cinco acuerdos, por tratar del programa intersectorial, y que, a su vez, dieron origen a otros documentos para ALC en lo que dice respecto a la protección y educación de los niños. En el Cuadro 2 se presentan, resumidas, las acciones instituidas. 
Cuadro 2. Principales acciones instituidas para la intersectorialidad (global-regional)

\begin{tabular}{|c|c|c|c|}
\hline & $\begin{array}{l}\text { Documento / } \\
\text { Compromiso }\end{array}$ & Coordinación & Elementos para la infancia \\
\hline \multirow{3}{*}{$\begin{array}{l}\bar{\pi} \\
\frac{0}{0} \\
0\end{array}$} & $\begin{array}{l}\text { Declaración Mundial } \\
\text { sobre Educación para } \\
\text { Todos (1990) }\end{array}$ & Unesco & $\begin{array}{l}\text { El aprendizaje comienza en el nacimiento; deman- } \\
\text { da atención y desarrollo en la primera infancia; } \\
\text { involucra familias, comunidades o programas } \\
\text { institucionales. }\end{array}$ \\
\hline & $\begin{array}{l}\text { Marco de Acción de } \\
\text { Dakar (2000) }\end{array}$ & Unesco & $\begin{array}{l}\text { Unir acciones del Estado, la familia y comunidad; } \\
\text { orientación en cuanto a que tanto los programas } \\
\text { formales como los no formales incluyeran la } \\
\text { Educación preescolar basada en la Atención y } \\
\text { Educación de la Primera Infancia (AEPI), sobre } \\
\text { todo de cero a los tres años, así como acciones, } \\
\text { políticas y programas para la infancia que abar- } \\
\text { casen los campos de la educación, salud y otros } \\
\text { aspectos biopsicosociales, todo coordinado de } \\
\text { forma multisectorial e intersectorial. }\end{array}$ \\
\hline & $\begin{array}{l}\text { Objetivos de Desarro- } \\
\text { Ilo del Milenio (ODM) } \\
\text { (2000) }\end{array}$ & $\begin{array}{l}\text { Organización } \\
\text { de las Nacio- } \\
\text { nes Unidas } \\
\text { (ONU) }\end{array}$ & $\begin{array}{l}\text { Objetivos que contemplan, en alguna medida, } \\
\text { a la infancia: erradicar la pobreza extrema y el } \\
\text { hambre; reducir la mortalidad infantil; mejorar la } \\
\text { salud materna; combatir el VIH/SIDA, la malaria } \\
\text { y otras enfermedades; desarrollar una alianza } \\
\text { global para el desarrollo. }\end{array}$ \\
\hline \multirow[t]{2}{*}{ 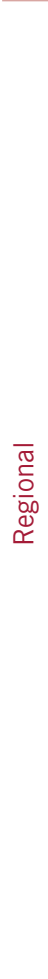 } & $\begin{array}{l}\text { VII Sesión del Comité } \\
\text { Intergubernamental } \\
\text { Regional del Pro- } \\
\text { yecto Principal para } \\
\text { la Educación (PRO- } \\
\text { MEDLAC VII), Cocha- } \\
\text { bamba (2001). }\end{array}$ & Unesco & $\begin{array}{l}\text { El cuidado y el desarrollo integral de la primera } \\
\text { infancia con enfoque centrado fundamentalmente } \\
\text { en la familia y en la generalización del acceso a } \\
\text { la educación inicial, que debe constituirse como } \\
\text { pilar fundamental de toda buena educación; am- } \\
\text { pliar la atención dirigida por la educación formal } \\
\text { y no formal a los grupos de niños pequeños para } \\
\text { mejorar la calidad y la equidad en el aprendizaje } \\
\text { a lo largo de toda la vida; cooperación interna- } \\
\text { cional renovada y nuevos actores; incrementar la } \\
\text { inversión social en la educación y el cuidado y } \\
\text { protección de la primera infancia, especialmente } \\
\text { de la población más vulnerable; oferta educativa } \\
\text { de } 3 \text { a } 6 \text { años y de servicios para los menores } \\
\text { de } 3 \text { años, con prácticas intersectoriales en el } \\
\text { campo de la educación; el Estado debe asumir un } \\
\text { liderazgo efectivo, estimulando la participación } \\
\text { de la sociedad en el planeamiento, ejecución y } \\
\text { evaluación de la investigación sobre el impacto } \\
\text { de las políticas educativas. }\end{array}$ \\
\hline & $\begin{array}{l}\text { Modelo de Acompaña- } \\
\text { miento: Apoyo, Moni- } \\
\text { toreo y Evaluación del } \\
\text { Proyecto Regional de } \\
\text { Educación para Amé- } \\
\text { rica Latina y el Caribe } \\
\text { (Prelac) (2002) }\end{array}$ & Unesco & $\begin{array}{l}\text { Acciones para la región: a) identificación de la } \\
\text { población de } 0 \text { a } 6 \text { años sin atención educativa; } \\
\text { b) preparación e implantación de programas } \\
\text { educativos con enfoque intersectorial y carácter } \\
\text { comunitario que incentiven el importante papel } \\
\text { de la familia; c) la educación en cuanto a as- } \\
\text { pectos de la salud y de la nutrición, así como el } \\
\text { enriquecimiento cultural. }\end{array}$ \\
\hline
\end{tabular}

Fuente: organizado por las autoras a partir de los documentos (2020). 
Al presentar los pactos, declaraciones internacionales y proyectos transnacionales incorporados por los Estados nacionales, patrocinados, apoyados y/o coordinados por los OI, ni olvidamos el poder de los Estados ni minimizamos su participación en ese proceso, visto que, aunque sufran influencias, al mismo tiempo son sus productores (Ball, 2001) y, como tales, incorporan las directrices instituidas en los compromisos internacionales con particularidades locales. En el caso de las orientaciones para la infancia, el panorama legal pone de manifiesto significativos cambios realizados en los países, sobre todo a partir de la Convención sobre los Derechos del Niño (1989) y de los compromisos firmados y declaraciones con los planes de acción nacionales:

1. Compromisos con la infancia desde la Constitución Política: Brasil (1988); Colombia (1991); Chile (1991-2000); Ecuador (2008); El Salvador (1993); Paraguay (1993); Perú (1993); Uruguay (2000); Venezuela (1999); México (2004).

2. Reformas educativas y Leyes Generales u orgánicas de educación que, en sus contenidos, contienen la esencia de la Convención sobre los Derechos del Niño: Argentina (1993); El Salvador (1968); Bolivia (1994); Brasil (1996); Chile (1990); Cuba (1991); Ecuador (2011); Granada (1980); Guatemala (1991); Paraguay (2005); Jamaica (2001); Trinidad y Tobago (1993); República Dominicana (1997); México (1993); Cuba (1982); Nicaragua (1979); Panamá (1994); Perú (1968); Uruguay (2000); Venezuela (1996).

Más allá de los documentos, los incentivos para estas nuevas políticas se reflejaron en el impacto de ideas y conocimientos compartidos (Pereira, Bernardo, Culpi y Pessali, 2018). En el caso de la intersectorialidad para la infancia, hubo una revisión, adaptación y transferencia de las políticas propuestas de acuerdo con las realidades e intereses nacionales, alineadas a los documentos orientadores internacionales, prácticas identificadas como de collage o convergencia de políticas (Ball, 2001).

\section{De la agenda de cooperación internacional a la policy transfer: la educación intersectorial no formal para la infancia}

Al revisar experiencias latinoamericanas y caribeñas que involucren políticas y programas intersectoriales, un estudio de Umayahara (2004) demuestra, como tendencia, la concreción de programas no formales recontextualizados, mediados por agencias internacionales, centros de investiga- 
ción, consultores y Estados de la región. El proceso se configura a partir de la policy transfer, favorecido por los procesos de gobernanza, comprendiendo una agenda de procesos, a través de los cuales el conocimiento sobre políticas, instituciones e ideas de una realidad, se utiliza en el desarrollo de políticas, normas administrativas, instituciones e ideas en otro escenario político. También implica el aprovechamiento de experiencias exitosas de otros contextos regionales y/o internacionales consideradas dignas de transferencia (Krawczyk, 2019). Dicha agenda se beneficia por la globalización y por la regionalización (Evans y Davies, 1999; Stone, 2004; Hassenteufel et al., 2017; Porto de Oliveira y Faria, 2017).

La transferencia debe entenderse dentro del concepto de recontextualización, pues, en cada país intervienen mecanismos de conformación con diferencias contextuales. La política se incorpora a partir de un modelo exitoso ya implantado por medio de transferencia de textos, prácticas y objetivos que pasan por modificaciones a lo largo del proceso de recontextualización de acuerdo con las especificidades de cada realidad local. Ese proceso puede moldear tanto el contenido como la forma del conocimiento compartido, pero no modifica la esencia ni la finalidad de la política transferida (Dolowitz y Marsh, 2000).

La transferencia recontextualizada se da de diferentes formas: gobernanza jerárquica, gobernanza negociada y gobernanza facilitada (Bulmer y Padgett, 2004). El modelo de gobernanza jerárquica trata de las reglas formales e informales por medio de la autoridad supranacional, impuesta sobre los gobiernos nacionales. En cambio, la gobernanza negociada se concreta en la transferencia por la adopción del modelo o de la política, cuyas reglas se establecen en la negociación política de los involucrados. Por último, la gobernanza facilitada, mantiene la soberanía nacional y las instituciones involucradas, moldeando los procesos de transferencia en políticas públicas (Bulmer y Padgett, 2004). Esos elementos pueden darse de forma aislada o en sinergia, en función de las nuevas relaciones establecidas entre el Estado y la sociedad, que posibilitan la flexibilización de la política de acuerdo con el pacto concertado para las prácticas de transferencia.

Al acordar el programa relativo a la educación para la infancia, la Unicef y la Unesco no solo actuaron como agentes de difusión en la gobernanza jerárquica, sino también como mediadores, planificadores, financiadores y consultores en la policy transfer, en calidad de sujeto de gobernanza negociada y facilitada. El modelo transferido seleccionado para análisis en este estudio fue el programa no formal cubano Educa a tu hijo, enfocado en la intersectorialidad para la infancia. Los criterios para su elección fueron: 
haber sido transferido y fomentado por los OI; atender al programa general internacional que conduce a programas no formales e intersectoriales; y, comprender acciones para la infancia en América Latina.

\subsection{El programa Educa a tu Hijo: transferencia de políticas en países de América Latina y el Caribe}

El programa cubano Educa a tu Hijo surgió como resultado de una investigación realizada en la década de 1970 por el Instituto Central de Ciencias Pedagógicas de Cuba, con el objetivo de ofrecer alternativas educativas a niños residentes en áreas rurales y montañosas, que no tenían acceso a los Círculos infantiles ${ }^{3}$. Entre 1983 y 1992 se desarrollaron programas piloto que, debido a sus buenos resultados, se implementaron como política de Estado en 1992. El programa analizado se propuso alcanzar el nivel máximo de desarrollo posible para cada niño en las áreas de comunicación emocional, inteligencia, lenguaje, desarrollo motor, formación de hábitos, salud y nutrición (Tinajero, 2010), involucrando a "la familia, la comunidad y el enfoque intersectorial” (Gómez, 2011, p. 18).

El referido programa es de responsabilidad del Ministerio de Educación cubano (Tinajero, 2010) y opera de acuerdo a un modelo no institucionalizado, intersectorial y de base comunitaria reconocido como programa social de atención educativa (Gómez, 2011). En el Cuadro 3 se presentan sus principales elementos.

Cuadro 3. Elementos del Programa Educa a tu Hijo

$\begin{gathered}\text { Participación de } \\ \text { la familia y de la } \\ \text { comunidad }\end{gathered}$
$\begin{gathered}\text { Prácticas de } \\ \text { desarrollo infantil }\end{gathered}$

Fuente: Adaptado por las autoras de Tinajero (2010).

${ }^{3}$ Son instituciones educativas formales destinadas a niños de 0 a 6 años. Los niños egresan de los círculos infantiles para ingresar a la escuela primaria. 
El programa integró los conceptos de la Convención sobre los Derechos del Niño (Unicef, 1989), en el sentido de exigir el cumplimiento del principio de la responsabilidad conjunta del Estado, la sociedad civil y la familia. Desde la creación del programa en Cuba, en 1992, la Unicef asumió el protagonismo con respecto a la educación inicial. Con relación al Educa a tu Hijo, cumplió un "rol fundamental en la implementación del programa, no sólo apoyando en el diseño de los materiales, sino también en todo el proceso de expansión y evaluación y siguiendo muy de cerca su desarrollo" (Gómez, 2010, p. 94). La Unicef se desdobló para que Cuba alcanzara los Objetivos de Desarrollo del Milenio (ODM), establecidos por la ONU, y cumpliera con los compromisos de educación para todos, acordados en Dakar (2000). Y, en efecto, con este programa, prácticamente se universalizó la educación inicial en Cuba.

En la Figura 1 se representa el período entre la institucionalización del programa Educa a tu Hijo y la evaluación de la Unesco sobre los resultados de la atención de la primera infancia en Cuba. El Informe de Seguimiento de la Educación para Todos en el Mundo (Unesco, 2015) señala a Cuba como el único país que alcanzó todos los objetivos mensurables de educación, con un $99,7 \%$ de atención.

Figura 1. Institucionalización del programa Educa a tu hijo y atención dirigida a la infancia cubana

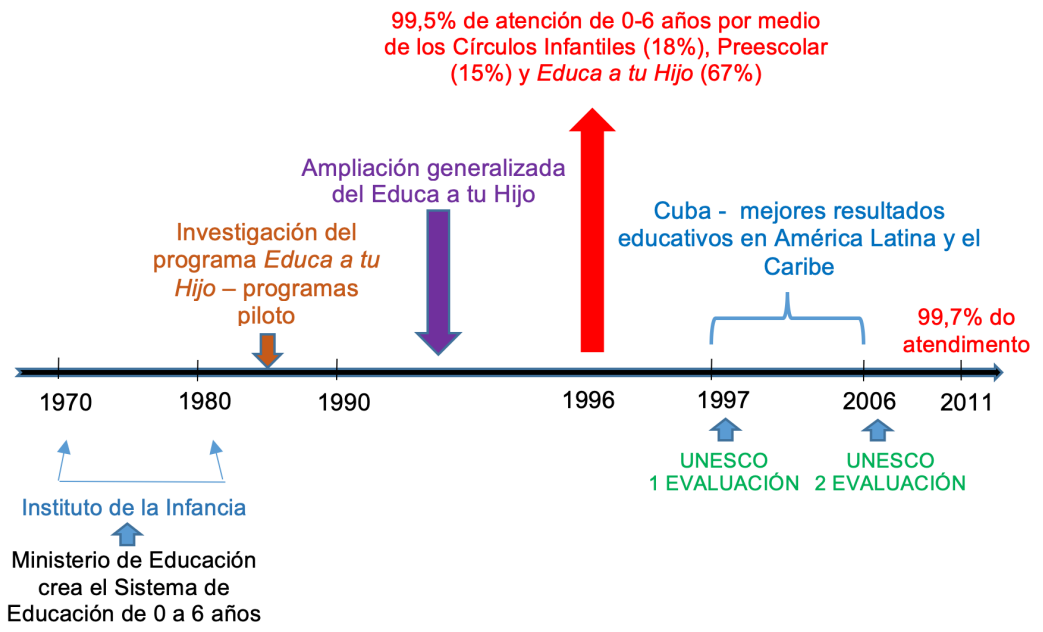

Fuente: elaborado por las autoras a partir de Cuba (1992; 2014). 
La Unicef y la Unesco, junto al Centro de Referencia Latinoamericano para la Educación Preescolar (CELEP) ${ }^{4}$, divulgaron y recontextualizaron el programa, tomándolo como referencia y punto de partida para la implantación de programas intersectoriales para la educación de la infancia en América Latina. Dicho proceso se inició a través de la presentación del programa cubano en foros nacionales e internacionales, plasmado en el documento de la oficina cubana de Unicef "La experiencia cubana en la atención integral al desarrollo infantil en edades tempranas" (2002), así como en el estudio de caso "Estudio nacional sobre la coordinación nacional de políticas y programas para la primera infancia" (2004), elaborado por la oficina de la Unesco en París. De esa forma, el programa atrajo la atención de especialistas de países de América Latina, que buscaron, ante el CELEP, informarse sobre el programa (Goméz, 2011).

EI CELEP ha llevado a otros países la experiencia cubana, la cual ha sido tomada como punto de partida para organizar programas similares al Educa a tu Hijo en contextos económicos, sociales y culturales distintos, porque es un modelo flexible y exitoso, cuando se cumplen adecuadamente las estrategias para el trabajo familiar, comunitario e intersectorial (Umayahara, 2004). Además, el documento de la Unesco de París, en su presentación, resalta:

Queremos compartir con todos la satisfacción de mostrar una experiencia exitosa, diez años después de iniciada, en un tema que constituye, para el gobierno de Cuba y la Unicef, una prioridad: la atención integral a la primera infancia (Unesco, 2002, traducción libre).

La transferencia contó con la asistencia técnica del Celep y de la Unesco y con el fomento de la Unicef, que lo vio como una posibilidad para la concreción de las metas acordadas en el programa internacional para la infancia. La metodología se reprodujo en Ecuador, Brasil, México, Colombia y Guatemala (Tinajero, 2010, p. 12) en un proceso que identificamos como policy transfer.

Imagen 2. Flujograma de la policy transfer del programa Educa a tu Hijo

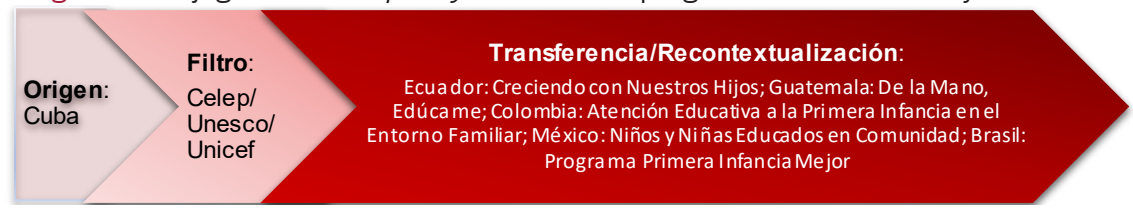

Fuente: Elaboración propia, 2020.

${ }^{4}$ Creado en 1997, con sede en Cuba, funciona como comisión social de investigación científica con el objetivo de coordinar los aspectos técnicos y científicos de las ciencias pedagógicas aplicadas a edades preescolares, ejecutar programas de enseñanza de posgrado, así como realizar intercambio científico con profesionales de los países de ALC vinculados a la educación de niños y niñas en la educación inicial. 
Para ello, se registró una colaboración sistemática de la Unicef en la edición de materiales, en la garantía de la capacitación de personal y en el apoyo a la investigación y recontextualización. Ecuador, Guatemala y Brasil contaron con la edición de la bibliografía vinculada a sus programas, con el apoyo financiero de las oficinas de Unicef ubicadas allí, así como con la asesoría cubana, por medio del CELEP, financiada por la Unicef (Tinajero, 2010).

Además del financiamiento, la Unesco ofreció cooperación técnica para el caso brasileño (por medio de la oficina de la Unesco en Brasil) y para el mexicano, contó con la consultoría de Marta Llanos en el proyecto "Mejoramiento de la calidad de la educación básica en el estado San Luis Potosí", en 2003 (Gómez; Morán y Beltrán; Ferreiro et al., 2011; Tinajero, 2010). En el Cuadro 4 se ponen en evidencia algunas características asumidas por el programa cubano en los países que realizaron su recontextualización.

Cuadro 4. Programas transferidos a partir del programa Educa a tu Hijo

\begin{tabular}{|c|c|c|c|c|}
\hline País & Estado o municipio & Nombre del programa & Inicio & Estado actual \\
\hline Ecuador & $\begin{array}{l}\text { Iniciado en Quito, } \\
\text { Cuenca, Manabí, } \\
\text { Chimborazo y Loja }\end{array}$ & $\begin{array}{l}\text { Creciendo con nuestros } \\
\text { hijos }\end{array}$ & 1997 & Ampliado a todo el país \\
\hline Guatemala & $\begin{array}{l}\text { Quetzalternango, } \\
\text { Chimaltenango }\end{array}$ & De la mano, edúcame & 2002 & $\begin{array}{l}\text { El cambio de gobierno en } \\
2004 \text { interrumpió su implan- } \\
\text { tación y el programa dejó de } \\
\text { operar en } 2006\end{array}$ \\
\hline
\end{tabular}

\begin{tabular}{|c|c|c|c|c|}
\hline Brasil & Rio Grande do Sul & $\begin{array}{l}\text { Programa Primera Infancia } \\
\text { Mejor }\end{array}$ & 2003 & $\begin{array}{l}\text { Ampliación en municipios } \\
\text { del estado }\end{array}$ \\
\hline Colombia & $\begin{array}{l}\text { Al principio, en An- } \\
\text { tioquia, Boyaca, La } \\
\text { Guajira y municipio } \\
\text { de Medellín }\end{array}$ & $\begin{array}{l}\text { Adoptó los nombres de los } \\
\text { programas de gobierno o } \\
\text { de los municipios en los } \\
\text { cuales se implantó, en } \\
\text { el ámbito de la política } \\
\text { de Atención Educativa a } \\
\text { la Primera Infancia en el } \\
\text { Entorno Familiar }\end{array}$ & 2007 & En proceso de ampliación \\
\hline \multirow[t]{5}{*}{ México } & San Luis Potosí & Educa a tu hijo & 1999 & $\begin{array}{l}\text { Con el cambio de gobierno, } \\
\text { se interrumpió su implan- } \\
\text { tación. }\end{array}$ \\
\hline & Oxaca & $\begin{array}{l}\text { Niños y niñas en comunidad } \\
\text { (NYNCEC) }\end{array}$ & 2003 & En proceso de ampliación \\
\hline & Michocan & De la mano con tu hijo & 2003 & $\begin{array}{l}\text { En regiones fundamen- } \\
\text { talmente rurales, hasta el } \\
\text { cambio degobiernoen } 2008\end{array}$ \\
\hline & Tabasco & $\begin{array}{l}\text { La educación no espera, } \\
\text { edúcame }\end{array}$ & 2003 & $\begin{array}{l}\text { El cambio de gobierno, en } \\
2009 \text {, dio término a su } \\
\text { implantación. }\end{array}$ \\
\hline & Nuevo León & Aprendiendo juntos & 2002 & $\begin{array}{l}\text { Orienta a las familias en los } \\
\text { centros de desarrollo infantil } \\
\text { (CENDIS) sin proceso de } \\
\text { visitas domiciliarias. }\end{array}$ \\
\hline
\end{tabular}

Fuente: organizado por las autoras a partir de Gómez (2011). 
En el Cuadro 5 se analizan las categorías de la policy transfer de los programas intersectoriales, con el fin de explicar cómo se llevaron a cabo las transferencias y cuáles fueron los límites de los programas recontextualizados.

Cuadro 5. Categorías de análisis de los programas intersectoriales destinados a la infancia transferidos en América Latina y el Caribe

\begin{tabular}{|c|c|}
\hline Preguntas & Categorías de análisis de transferencia \\
\hline $\begin{array}{l}\text { ¿Cómo se involucran los } \\
\text { actores en la policy transfer? }\end{array}$ & $\begin{array}{l}\text { Por medio de transferencia voluntaria y transferencia coercitiva, } \\
\text { a partir de gobernanza negociada y gobernanza facilitada. }\end{array}$ \\
\hline $\begin{array}{l}\text { ¿Quiénesson los actoresclave } \\
\text { involucrados en el proceso? }\end{array}$ & $\begin{array}{l}\text { Políticos electos; partidos políticos, burócratas/empleados públi- } \\
\text { cos, grupos de presión, emprendedores, corporaciones transna- } \\
\text { cionales, instituciones gubernamentales y no gubernamentales } \\
\text { supranacionales y consultores. }\end{array}$ \\
\hline ¿Qué se transfiere? & $\begin{array}{l}\text { Los objetivos de la política; instrumentos de la política; contenido } \\
\text { de la política; programas de la política; instituciones, ideologías; } \\
\text { ideales, actitudes y conceptos; lecciones negativas. }\end{array}$ \\
\hline $\begin{array}{l}\text { ¿Desde dónde se diseñan las } \\
\text { lecciones? }\end{array}$ & Gobernanza: nacional, local e internacional. \\
\hline $\begin{array}{l}\text { ¿Cuáles son los diferentes } \\
\text { grados de transferencia? }\end{array}$ & Copia, emulación, combinaciones e inspiración. \\
\hline Límites de esta policy transfer & $\begin{array}{l}\text { Focalización, discontinuidades, variados arreglos de atención } \\
\text { que pueden comprometer la calidad esperada. }\end{array}$ \\
\hline
\end{tabular}

Fuente: adaptado por las autoras a partir de Dolowitz y Marsh (2000).

El análisis de la documentación de los países permitió identificar que todos los programas implantados atendían a la lógica de la educación no formal y que revelaban afinidad con el contenido de las declaraciones en las convenciones internacionales, focalizando en la ampliación de la atención a la infancia acordada por los Estados signatarios.

Se observó, además, la tendencia a la descentralización y a la focalización en los países que transfirieron el programa. Según Campos (2012), la adopción de este tipo de estrategia acaba por promover nuevas formas de segmentación y de focalización de políticas que, lejos de distribuir con "equidad" las "oportunidades educativas", crean nuevas formas de discriminación y de subalternización (Campos, 2012; Bortot y Lara, 2019; Moreira, 2019).

Por otra parte, se observó que en Guatemala y México (en algunos municipios) interrumpieron el desarrollo del programa, poniendo de manifiesto, así, un proceso de discontinuidad. La discontinuidad de las políticas públicas educativas se relaciona con la fragmentación de acciones públicas, debido a los procesos sucesorios, articulaciones políticas y rupturas en el proceso de financiamiento de la educación. Por tanto, no fue posible identificar políticas de Estado que buscasen cambiar la realidad del niño y de sus familias, y que dieran lugar a cambios de otras normas o disposiciones preexistentes, 
con incidencia en sectores más amplios de la sociedad (Oliveira, 2011), sino políticas de gobierno, ya sea en el ámbito nacional, estadual o municipal, con determinadas medidas y programas con los cuales se buscaba atender a las demandas de la agenda mundial/regional.

Se registró también, en los casos analizados, la flexibilización de la política por la cual el objetivo se mantuvo, así como los principales ejes, pero las estrategias de acción se modificaron. En Colombia, el programa, así como toda la política para la primera infancia, se orientó hacia alianzas con el sector privado. En Ecuador, el modelo lo implementó una agencia privada sostenida con financiamiento público, el Instituto Nacional del Niño y la Familia (INNFA), ya extinto y hoy transformado en una entidad de derecho público. Actualmente, en esos países, la coparticipación y corresponsabilidad del Estado y de la sociedad civil reposan sobre las políticas de atención a la infancia, en diferentes sectores y asociaciones civiles (Tinajero, 2010).

En el caso brasileño, el programa Primera Infancia Mejor (PIM) tuvo dos ejes centrales en su puesta en marcha: la descentralización y las alianzas público-privadas. La ley que instituyó el $\mathrm{PIM}^{5}$, indica, en su art. $3^{\circ}$, que el Programa:

[...] se implantará en todos los municipios con la colaboración de los sectores responsables por las áreas de educación, salud y asistencia social y de organizaciones no gubernamentales, de programas de orientación y apoyo a los padres con hijos de 0 a 3 años" (Estado do Rio Grande do Sul, 2006, p. 1).

En el art. $7^{\circ}$, la descentralización de la acción se señaló a partir de la garantía de su ejecución:

EI PIM será ejecutado por los municipios o por organizaciones no gubernamentales mediante Pliego de Adhesión" (Estado do Rio Grande do Sul, 2006, p. 3).

La materialización de la agenda se hizo, predominantemente, por medio de la policy transfer, asegurada por la gobernanza facilitada, pues los Estados establecen intercambio de información y conocimientos, pero no son impelidos a transferir la política en su totalidad. Así, los Estados nacionales mantienen su soberanía adecuando los programas a sus leyes orgánicas y transfiriendo solo los aspectos que consideran ventajosos (Pereira et al., 2018).

\footnotetext{
${ }^{5}$ Actual Ley $n^{\circ} 14.594 / 2014$
} 
Las diferencias esenciales con relación al programa original cubano están en la puesta en marcha del programa. Por ejemplo, se constató que el foco estuviera en la familia y no en el niño, como se puso de manifiesto en el caso de Ecuador, así como la articulación e interrelación con diferentes sectores privados para la ejecución del programa, como se observó en Brasil y Ecuador.

Además de políticas de transferencia orientadas por los Ol dentro del programa regional, se identificó la cooperación técnica en el planeamiento, la organización de material, la capacitación, la asesoría, la evaluación, actuando dentro de la gobernanza negociada y facilitada. En la implantación de las acciones intersectoriales, se observaron características de la gobernanza ampliada, realizada por medio del incentivo y de la atención, mediante los diversos acuerdos educativos, como las ONG, el Tercer Sector, asignación de partidas públicas y colaboración con el sector privado, como corrobora Pereira (2019) en un estudio realizado sobre la OCDE, cuyo principio de gobernanza presupone ampliar la:

[...] participación de sectores de la sociedad en la definición y ejecución de las políticas públicas [en las] que [...] se destacan los sectores privados, que pasan no solo a definir cuáles son las prioridades de los sistemas educativos, así como su contenido y la formación de los docentes, sino también la ejecución de políticas (p. 1.730).

Desde esa perspectiva, el Estado no es, necesariamente, aquel que concede financiamiento para el programa, papel asignado a las instituciones privadas, organizaciones no gubernamentales y sociedad civil. De esa forma, se retira toda carga del gobierno siempre y cuando se ejerza su función descentralizadora y concentradora de políticas (Ferreira, Fonseca y Scaff, 2019). Para esa base de organización social, bastaría la mínima garantía de derechos provenientes del Estado nacional destinados a los más vulnerables.

Las políticas analizadas ponen en evidencia una convergencia de paradigmas de orden económico, como: descentralización, programas de bajo costo, atención no formal como alternativa a la escolar, agenda internacional, flexibilización, carácter compensatorio, alianzas público-privadas para la atención y discontinuidades en el desarrollo de la política.

\section{Consideraciones finales}

El estudio llevado a cabo nos permitió constatar que los Ol actuaron como órganos de gobernanza regional en lo relativo a las políticas para la infancia en países de ALC. Que la Unesco y la Unicef adquirieron una 
gran capacidad como agencias en la región, y esa intervención les hizo ser agentes de gobernanza para la educación de la infancia, que construyen, legitiman y operan las redes transnacionales y comunidades epistémicas para la educación; elaborando documentos de evaluación y acordando junto a los países metas y plazos; instituyendo programas internacionales con estrategias intersectoriales; reconociendo políticas de éxito y fomentando su transferencia a otros contextos políticos; certificando las políticas que comportan el reconocimiento por parte de una autoridad externa; apoyando, distinguiendo y legitimando las prácticas, como en el caso del modelo cubano; asesorando a los países en los procesos de difusión de políticas; realizando funciones operativas de asistencia y en la generación de ideas; financiando proyectos y subsidiando las asesorías.

La educación intersectorial se reveló como una estrategia de gobernanza en la que las experiencias de una comunidad educativa "ampliada" son vastamente puestas de relieve por los Ol y absorbidas por los países, sirviéndose de su legitimidad y pericia en cuanto a la gobernanza transnacional de la Educación, como señalan Gorostiaga (2020) y Shiroma (2020). Las acciones priorizadas en el contexto analizado correspondieron a programas no formales y se organizaron por medio de la policy transfer, cuyo foco de gobernanza busca diversificar los actores sociales que actúan en el campo de las políticas públicas.

Estas nuevas formas de vinculación entre sociedad y Estado impactaron en la construcción prioritaria de políticas educativas y abordajes de reformas, que al cumplir el programa internacional de educación y pobreza para la infancia, expandienron las formas de atención y tuvieron en cuenta el área productiva futura de los niños y de sus familias. En definituva, los países se apropiaron de esas acciones intersectoriales en sus políticas, flexibilizándolas en propuestas focales, locales, descentralizadas y, como se ha constatado, aunque marcadas por discontinuidades, a diferencia de la experiencia cubana cuyo programa se desarrolla de forma continua, registraron mejoras atendiendo a las necesidades y expectativas de la comunidad involucrada.

\section{Bibliografia}

Ball, S. (2001). Diretrizes Políticas Globais e Relações Políticas Locais em Educação. Currículo sem Fronteiras, 1(2), 99-116.

Ball, S. (2007). Understanding private sector participation in public sector education. Routledge: London. 
Ball, S. (2014). Educação Global S. A.: novas redes de políticas e o imaginário neoliberal. Tradução de Janete Bridon. Ponta Grossa: UEPG.

Blanco, R. y Umayahara, M. (2004). Síntesis regional de indicadores de la primera infancia. Santiago de Chile: Oficina Regional de Educación para América Latina y el Caribe OREALC/UNESCO.

Bortot, C. (2018). Atores e autores de políticas para a educação infantil de zero a três anos no Brasil: transformação do Estado e das suas relações com a sociedade civil (20002015). 255f. Dissertação (Mestrado em Educação) - Universidade Estadual de Maringá, Brasil.

Bortot, C. y Lara, A. M. B. (2019). As políticas de Educação e Cuidados na Primeira Infância para a América Latina: intencionalidades e encaminhamentos na proposta da Unesco. Revista Ibero-Americana de Estudos Em Educação, 14, 1767-1781.

Bulmer, S. y Padgett, S. (2004). Policy transfer in the European Union: an institutionalist perspective. British Journal of Political Science, 35, 103-126.

Campos, R. F. (2012). "Política pequena" para as crianças pequenas? Experiências e desafios no atendimento das crianças de 0 a 3 anos na América Latina. Revista Brasileira de Educação, 17(49).

Casassus, J. (2001). A reforma educacional na América Latina no contexto de globalização. Cadernos de Pesquisa, 114, 7-28.

Casula, M (2017). Quem governa na governança (local)? Reflexões teóricas e evidências empíricas, Revista de Administração Pública, 51(6).

Costa, M. O. (2019) Contrarreformas, Nova Gestão Pública e relações público- privadas: mapeando conceitos, tendências e influências na educação, RBPAE, 35(1), 159-179.

Cowen, R. (2006). Acting comparatively upon the educational world: puzzles and possibilities. Oxford Review of Education, 32(5), 561-573.

Cuba (1992). Ministerio de Educación. Educa a tu hijo. Programa para la familia dirigido al desarrollo integral del niño. La Habana: Editorial Pueblo y Educación.

Cuba. (2014). Educación para todos: Reporte de la Revisión Nacional de Educación para Todos al 2015 de la República de Cuba.

Dolowitz, D. P. y Marsh, D. (2000). Learning from abroad: the role of policy transfer in contemporary policy-making. Governance: an international journal of policy and administration, 13(1), 5-24.

Estado do Rio Grande do Sul. (2006). Lei n. 12.544, de 03 de julho de 2006. (Atualizada ate a lei n. 14.594, de 28 de agosto de 2014). Institui o Programa Primeira Infância Melhor - PIM - e dá outras providências.

Evans, M. y Davies, J. (1999). Understanding policy transfer: a multi-level, multi-disciplinary perspective. Public Administration, 77(2), 361-385.

Ferreira, E. B., Fonseca, M. y Scaff, E. A. S. (2019). A interferência do Banco Mundial no ensino secundário brasileiro: experiências históricas e desafios atuais. Revista Ibero-Americana de Estudos em Educação, 14(3), 1733-1749.

Ferreiro, R. I., Gómez, A. M. S., Sterling, H. Y., Móran, M. P. y Beltrán, M. T. B. (2011). Educa a tu hijo: la experiencia cubana en la atencion integral al desarrollo infantil en edades tempranas. Mined/Unicef/Celep. 
Gómez, A. M. S. (2011). La contextualización del Modelo de Atención Educativa no Institucional Cubano "Educa a tu Hijo" en Países Latinoamericanos. UNICEF/OEI.

Gómez, A. M. S., Morán, M. P y Beltrán, M. T. B. (2011). El modelo de atención no institucional "Educa a tu hijo": su contextualización en países latino-americanos. Editor Educación Cubana. Ministerio de Educación.

Gorostiaga, J. (2020). ¿Hacia la regulación postburocrática de los sistemas educativos latinoamericanos? Un análisis del discurso de los organismos multilaterales de la región en el período 2012-2018. Educar em Revista, 36.

Harvey, D. (2008). O neoliberalismo: história e implicações. São Paulo: Loyola.

Hassenteufel, P., Benamouzig, D., Minonzio, J. y Robelet, M. (2017). Policy diffusion and translation: the case of evidence-based health agencies in Europe. Novos estudos CEBRAP, 36(1), 77-96.

Ivo, A. B. L. (2002). Governabilidade e Governança na América Latina: teses e paradoxos. In: VII Congreso del CLAD, 2002, Lisboa. VII Congreso del CLAD, 1. 1-20.

Kissler, L. y Heidemann, F.G. (2006). Governança pública: novo modelo regulatório para as relações entre Estado, mercado e sociedade? Revista de Administração Pública, 40(3), 479-99.

Krawczyk, N. (2019). A política educacional e seus desafios na pesquisa: o caso do Brasil. Revista de Estudios Teóricos y Epistemológicos en Política Educativa, 4, 1-9.

López, N. y D’Alessandre, V. (2015). Políticas públicas para la primera infancia en América Latina. Reflexiones a 25 años de la ratificación de la Convención Internacional sobre los Derechos del Niño. Disponible en https://bit.ly/3ciqt5P

Moreira, J. A. S. (2019). Políticas para educação infantil e a agenda 2030 no Brasil Rev. FAEEBA, 28(54), 77-96.

Oliveira, D. A. (2011). Das políticas de governo à política de Estado: reflexões sobre a atual agenda educacional brasileira. Educ. Soc., 32(115), 323-337.

Onu. (2000). Millennium Development Goals. Disponível em: https://bit.ly/3bsFn8A.

Pereira. R. da S. (2019). Proposições da OCDE para América Latina: o PISA como instrumento de padronização da educação. Revista Ibero-Americana de Estudos em Educação, 14(3), 1717-1732.

Pereira, A. E., Bernardo, G. J., Culpi, L. A. y Pessali, H. F. (2018). A governança facilitada no Mercosul: transferência de políticase integração nas áreas de eduçação, migração e saúde. Revista de Administração Pública, 52, 285-302.

Porto de Oliveira, O. y Faria, C. A. P. (2017). Policy transfer, diffusion and circulation. Research Traditions and the state of the discipline in Brazil. Novos Estudos Cebrap, 36(1), 13-32.

Rhodes, A. W. (2012). Waves of governance. In: Levi-Faur, D. The Oxford handbook of governance, (pp. 33-48). Oxford: Oxford University Press.

Robertson, S.L. y Dale, R. (2017). Comparing Policies in a Globalizing World: methodological reflections. Educação \& Realidade, 42(3), 859-875. 
Robertson, S.L. y Verger, A. (2012). Governing Education Through Public Private Partnerships. In S. Robertson, K. Mundy, A. Verger \& F. Menashy (ed.). Public Private Partnerships in Education: New Actors and Modes of Governance in a Globalizing World, (pp. 21-42). Cheltenham: Edward Elgar.

Shiroma, E. (2020). Redes, experts e a internacionalização de políticas educacionais. Revista de Estudios Teóricos y Epistemológicos en Política Educativa, 5, 1-22.

Scaff, E. A. S. (2017). Estado, Mercado e Educação: Arranjos e tendências. Revista Educação Unisinos, 21, 146-154.

Stone, D. (2004). Transfer agents and global networks in the 'transnationalization' of policy. Journal of European Public Policy, 11(3), 545-566.

Tinajero, A. R. (2010). Ampliación de los servicios de desarrollo infantil temprano en Cuba - Programa Educa a Tu Hijo de Cuba: Estrategias y lecciones de su proceso de ampliación de cobertura, Wolfensohn Center for Development, Documento de Trabajo 16. Washington, DC: Brookings Institution.

Umayahara, M. (2004). Coordinación Intersectorial de Políticas y Programas de la Primera Infancia: Experiencias en América Latina. Oficina Regional de Educación para América Latina y el Caribe OREALC/UNESCO Santiago.

Unesco (2000). The Dakar Framework for Action. Paris: Graphoprint

Unesco (2001). Declaración de Cochabamba. PROMEDLAC VII, Cochabamba.

Unesco (2002a). Modelo de acompañamiento - apoyo, monitoreo y evaluación para América Latina y el Caribe. Habana.

Unesco (2002b). Proyecto regional de educación para América Latina y el Caribe (PROLAC). La Habana: UNESDOC. Disponible en https://bit.ly/2WjDEOW

Unesco (2004). Estudio nacional sobre coordinación de programas para la primera infancia. París: Unesco.

Unesco (2007). Informe de Seguimiento de la EPT en el Mundo. Bases sólidas: Atención y educación de la Primera Infancia. París: UNESCO. Unesco/OREALC: Paris.

Unesco (2015). Education for All 2000-2015: achievements and challenges; EFA Global Monitoring Report.

Unesco/Orealc. (2004a). Participación de las familias en la Educación Infantil Latinoamericana. Santiago, Chile.

Unicef (1989). Declaração Universal dos Direitos das Crianças. Disponível em: https://bit. |y/2AatMx|

Verger, A. y Normand, N. (2015). Nueva gestión pública y educación: elementos teóricos y conceptuales para el estudio de un modelo de reforma educativa global. Educação \& Sociedade, 36(32), 599-622. 